\title{
Performance Evaluation of LEACH, PEGASIS and TEEN Routing Protocols in Wireless Sensor Networks based on Network Lifetime
}

\author{
Hebron Hailu Wodajo ${ }^{1}$, K S Ananda Kumar ${ }^{2 *}$, Girmay Tekle Kidane ${ }^{3}$, Sisay Muleta Hababa ${ }^{4}$ \\ School of Computing \& Informatics, College of Engineering \& Technology, Dilla University, Dilla, Ethiopia \\ Email*: anandgdk@gmail.com
}

\begin{abstract}
With the advancement of technology in communication and electronic components, cheap and tiny microprocessors that are used to create wireless networks are becoming more readily available. The primary goal of this work is to compare the performance of various routing protocols chosen from wireless sensor networks (WSN) based on network lifetime. To do this, three well-known energy saving hierarchical routing protocols called Low Energy Adaptive Clustering Hierarchy (LEACH), Power-Efficient Gathering in Sensor Information Systems (PEGASIS) and Threshold Sensitive Energy Efficient Sensor Network Protocol (TEEN) were selected for the study. Because these network protocols rely on hierarchical structures to function and save energy, they are fascinating to investigate. In this research, four-performance metrics considered namely number of nodes in alive, average energy dissipated, operating nodes per round and network lifetime. As a result, the researcher conducted a general analysis of the protocols in terms of performance metrics. The findings of the study show that among the three routing protocols, PEGASIS has longer network lifetime and lesser power consumption while LEACH has the least network lifetime and the highest power consumption. On the other hand, TEEN has been figured out that it has medium performance in terms of both power consumption and network lifetime. This shows that LEACH is ideal for small networks and can be preferable during critical times. Whereas, PEGASIS is ideal for large networks as it has efficient power consumption which results in longer network lifetime.
\end{abstract}

Keywords - Network lifetime, Performance, Routing protocol, Wireless sensor networks.

\section{INTRODUCTION}

A wireless sensor network (WSN) is composed of lowpower sensor source nodes, a set of computation resources at the center to handle data aggregation, and a sink node where all information is collected at the end. Wireless sensor networks are used for a variety of monitoring applications. Some of the applications include environmental, health, military, home, and industrial automation. Routing is a technique for determining the best path between source and destination nodes [1].

Current research in the field of wireless sensor networks shows that no special consideration has been given to the time criticality of the target applications. Most current protocols assume that a sensor network is constantly collecting data from its surroundings or responding to a specific query. Networks that are intended to adapt quickly to changes in sensed qualities are required. That sensor networks should enable end users to manage the trade-off between energy efficiency, accuracy, and response times on a real-time basis. As a result, the goal of this study was to create a communication protocol that could match these requirements. The energy available to sensor nodes in wireless sensor networks is restricted. In other cases, recharging sensor nodes is impossible since they are installed in areas that are inaccessible to humans. As a result, replacing the batteries in these sensors becomes extremely difficult for humans [2,7].
The main objective of this work is on the evaluation of the three hierarchical routing protocols based on network lifetime and energy saving routing protocols which conserves system total energy in WSN. To look into the efficiency of power consumption of LEACH, PEGASIS and TEEN protocols in wireless sensor networks. To analyze the performance of LEACH, PEGASIS and TEEN protocol in wireless sensor networks with regard to network lifetime. To figure out the time to apply the hierarchal routing protocols to improve the network lifetime.

From the perspective of power consumption and extending the lifespan of WSN the researcher chose to study hierarchical routing protocols specifically, and analyzed in detail the three well-known power saving hierarchical routing protocols called LEACH, PEGASIS and TEEN protocol[3].

\section{RELATED WORK}

The optimum match for network scalability and effective communication is hierarchical-based routing. Routing protocols determine how the sensor nodes in a wireless sensor network will communicate with one another. The transmission of information signals consumes a greater amount of energy from sensor nodes in the network. The sensor network is partitioned into various groups known as clusters in clustering techniques for Wireless Sensor Networks. The sensor node chooses the cluster head, 
which is one of the group's nodes. The job of cluster head might rotate among all other cluster nodes, depending on the routing protocol's technique. The cluster head for this round is the sensor node, which delivers signal packets to the base station. The cluster head may perform data aggregation on the message before sending it to the base station [4].

In wireless sensor networks, Low Energy Adaptive Clustering Hierarchy (LEACH) is a TDMA-based MAC technology that includes clustering and a basic routing mechanism. Heinzelman et al. [1, 4] introduced Low Energy Adaptive Clustering Hierarchy as a hierarchical clustering approach for sensor networks (LEACH). LEACH's goal is to save energy by reducing the amount of energy required to build and maintain clusters, hence extending the life of a wireless sensor network. This energy-efficient method provides a number of advantages, including extending system lifetime and increasing network coverage [5].

The setup phase and the steady state phase are the two phases of LEACH's operation. A fixed fraction of nodes, $\mathrm{p}$, elect themselves as $\mathrm{CHs}$ as follows during the setup phase. A sensor node selects a random value between 0 and 1 called $r$. The node becomes a cluster-head for the current round if this random number is smaller than a threshold value, T (n). The threshold value, denoted by G, is determined using an equation that includes the required percentage to become a cluster-head, the current round, and the set of nodes that have not been picked as a clusterhead in the previous $(1 / \mathrm{P})$ rounds.

$$
T(n)=\frac{p}{1-p\left(\operatorname{rmod}\left(\frac{1}{p}\right)\right)} \text { if } n \in G
$$

The number of nodes involved in the $\mathrm{CH}$ election is given by $\mathrm{G}$. Each newly elected $\mathrm{CH}$ sends an advertisement message to the network's remaining new cluster-heads [6]. An improvement to the LEACH methodology was proposed in [2]. PEGASIS (Power-Efficient Gathering in Sensor Information Systems) is a chain-based protocol that is near optimum. The protocol's main principle is that nodes only need to connect with their closest neighbours in order to extend network lifetime, and they take turns talking with the base station. When the round of all nodes connecting with the base station is completed, a new round begins, and so on. Because the power draining is distributed evenly across all nodes, the power required to transmit data per round is reduced [6]. As a result, PEGASIS has two primary objectives. To begin, utilize collaborative tactics to extend each node's lifetime, resulting in a longer network lifetime. Second, to keep communication bandwidth utilization to a minimum, only enable local coordination between nodes that are close together.

TEEN (Threshold Sensitive Energy Efficient Sensor Network Protocol) [3] is a reactive wireless sensor network routing protocol that employs a clustering strategy similar to $\mathrm{LEACH}$. In the process of constructing a cluster, the TEEN protocol provides two hard and soft criteria, minimizing the amount of data transport through the use of a filter. Following the selection of the cluster head, two threshold parameters, as well as attempter data, should be broadcast through TDMA.

Hamdy H. El-Sayed et. al discussed about the LEACH, DEEC, TEEN and SEP protocols, performance comparison of throughput, PDR, dead nodes, alive nodes and overhead are calculated. Network lifetime also different for different protocols [9].

Sahar Alsafi et. al discussed about the DEEC, DDEEC, EDEEC and TDEEC Protocols, TDEEC improves stability and energy efficacy. TDEEC performs better as compared to DEEC, DDEEC and EDEEC in heterogeneous environment for wireless sensor networks [10].

\section{METHODOLOGY}

WSN architecture should include energy economy, fault tolerance, and network lifetime because a high number of battery-driven nodes are deployed in a WSN [1]. In an emergency, however, important information must be transferred as quickly and reliably as possible, therefore network speed and lifetime are top priorities. As a result, a WSN architecture that meets requirements both in regular and emergency situations is required. Many outstanding studies on data gathering systems that can be used in everyday settings have been published. A strategy to incorporating mechanisms for urgent information transfer into any well-designed data collection scheme for application-oriented communication. In the normal circumstance, this indicates that a WSN uses a data collection technique. When an emergency arises, a set of steps are taken to transmit critical information to the BS.

\section{A. LEACH Algorithm}

Basically, the development of LEACH protocol [8] is referring of the flowchart shown in fig. 1.

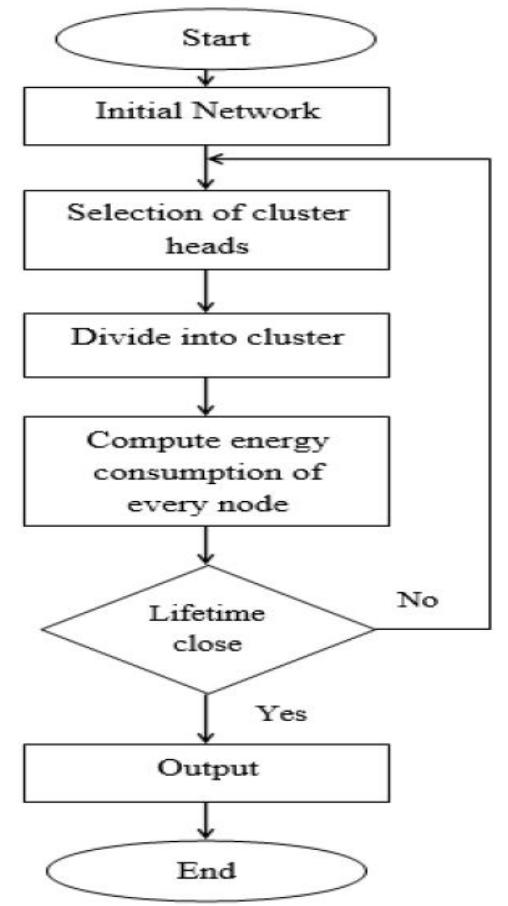

Fig.1. Flowchart of Leach Protocol 
The cluster formation and the cluster head selection algorithm are given below:

Step 1: Initialization is the first step.

Step 2: Process node i from step 3 to step 7 if it is $\mathrm{CH}$. If not, go back to step 1 .

Step 3: Using the CSMA MAC protocol, $\mathrm{CH}$ broadcasts an advertisement message (ADV). $\mathrm{ADV}$ is equal to the node's ID plus a distinguishing header.

Step 4: Each non-Cluster Head node decides its Cluster Head for this round based on the received signal strength of the ADV message.

Step 5: Using the CSMA MAC protocol, each non-Cluster Head sends a join-request message (Join-REQ) to its chosen Cluster Head. Join-REQ is equal to the node's ID plus the cluster-head ID plus the header.

Step 6: The Cluster Head node creates a TDMA schedule to coordinate data transfer within the cluster.

Step 7: TDMA schedule (1. Prevent data message collisions). 2. Non-cluster head node energy conservation).

Step 8: End.

\section{B. PEGASIS Algorithm}

PEGASIS' basic premise is that each node receives from and transmits to close neighbors, and that each node takes turns being the leader for transmission to the base station (BS). The energy burden will be distributed equitably among the sensor nodes in the network using this method, figure 2 shows the flowchart of PEGASIS protocol [2].

1. Initially, position the nodes in the play field at random.

2. The $i^{\text {th }}$ node is in an arbitrary position.

3. The nodes will be linked together to form a chain, which can be done by the sensor nodes themselves or by employing a greedy algorithm that starts at some node. The BS can also compute this chain and send it to all of the sensor nodes.

4. Use the greedy approach to build the chain, assuming that all nodes have global knowledge of the network.

5. The greedy method to chain construction is effective, and it is completed before the first round of communication.

6. To build the chain, start with the node that is farthest away from the BS. We start with this node to ensure that nodes further away from the BS have close neighbors, as the greedy approach would steadily increase neighbor distances because nodes already on the chain cannot be revisited.

7. Each node receives data from one neighbor in each round, fuses it with its own data, and transmits it to the next neighbor in the chain.

8. Node $\mathrm{i}$ will be in position $\mathrm{j}$ in the chain at random. Nodes take turns sending to the BS, and in round $\mathrm{i}$ we will utilize node number $\mathrm{i} \bmod \mathrm{N}$ ( $\mathrm{N}$ representing the number of nodes) to send data to the BS.

9. To make the sensor network resilient to failures, the leader in each cycle of transmission will be at random locations.

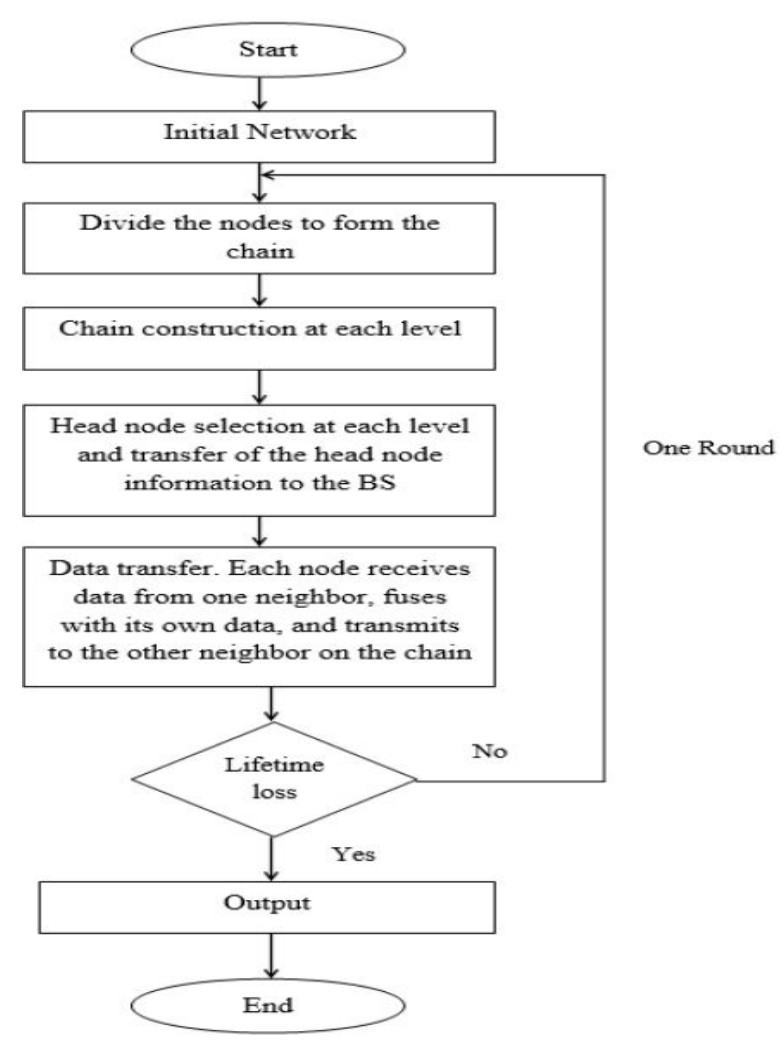

Fig.2. Flowchart of PEGASIS protocol

\section{TEEN Algorithm}

TEEN, a reactive clustering routing protocol, is improved by LEACH. The cluster head $(\mathrm{CH})$ of each cluster collects data from its members. Before transferring data to the BS or a higher-level $\mathrm{CH}$, the $\mathrm{CHs}$ integrate and process it. Clustering routing methods have the advantage that all nodes only need to convey data to their $\mathrm{CH}$, and only the $\mathrm{CHs}$ need to aggregate data. It helps to save electricity [3].

Each node takes turns as the $\mathrm{CH}$ to ensure that energy consumption is divided evenly. In the $\mathrm{CH}$ election, a random selection technique is used. Following the formation of clusters, $\mathrm{CHs}$ assign cluster members a time window during which they can submit data. In the TEEN routing system, unlike LEACH, CHs broadcast HT (Hard threshold) and ST (soft threshold) to their members to control the amount of data transferred. The nodes will only communicate sensed data to $\mathrm{CH}$ in the current round if the current value of the sensed attribute is greater than the value of HT.

The detected property is then saved in the internal variable sensed value (SV). Nodes constantly sense the attribute; it is only conveyed when the next value departs from SV by an amount equal to or greater than the ST. By discarding minimal change of detected property, the ST minimizes 
the frequency of data transmission. The value of ST can be adjusted to meet the needs of the users. Setting a smaller ST improves network accuracy at the expense of higher energy usage. As a result, users must control the trade-off between energy efficiency and precision by adjusting the size of ST. Figure 3 shows the flowchart for TEEN algorithm.

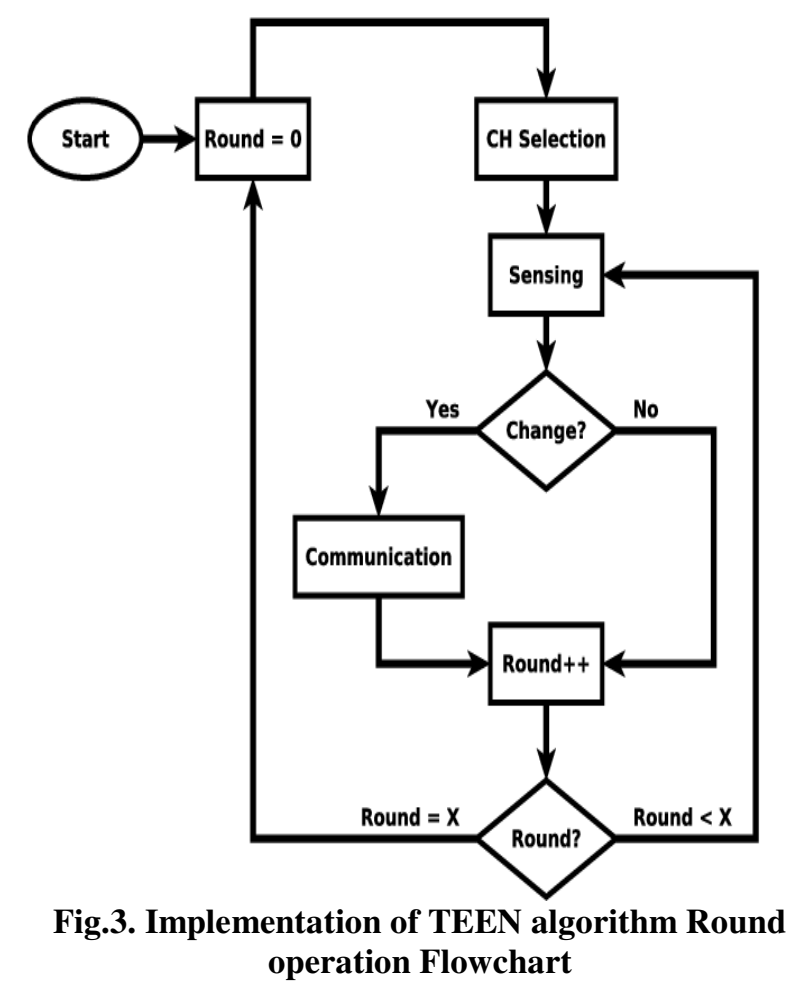

\section{RESULTS AND DISCUSSION}

To evaluate the performance of the hierarchal routing protocols simulated protocols performance using MATLAB simulator and compared the performance of LEACH, TEEN, and PEGASIS protocols on the basis of energy dissipation and the longevity of the network. The simulation consists of 100 homogeneous nodes and a fixed base station with initial energy of 2 joules, scattered randomly within a 100x100 unit's sensor field. Deployed nodes have fixed positions during the entire simulation.

\section{A. Number of Nodes alive}

The analysis was carried out in order to assess the effectiveness of these treatments. For the verification of these protocols' performance, there are a few things to keep in mind. The scenario had been executed in terms of dead nodes and the number of sensor node pathways. Figure 4 depicts the number of live nodes for LEACH, TEEN, and PEGASIS, where the $\mathrm{x}$-axis represents the number of rounds and the $y$-axis represents the number of dead nodes. The data values for the alive nodes of LEACH, TEEN, and PEGASIS methods are shown in Table 1.

TEEN is highly suited for time-critical applications and is energy efficient and quick to respond. It also enables the user to tailor the energy usage and precision to the application. For the $100 \mathrm{~m} \times 100 \mathrm{~m}$ network scenario, this graph displays the number of nodes that remain alive versus the number of activity. PEGASIS keeps all nodes alive for 400 seconds, whereas LEACH, TEEN, and PEGASIS keep nodes alive for 10, 40, and 100 seconds, respectively.

\section{TABLE 1 NUMBER OF NODES ALIVE}

\begin{tabular}{|l|l|l|l|}
\hline $\begin{array}{l}\text { Nodes } \\
\text { Alive }\end{array}$ & LEACH & TEEN & PEGASIS \\
\hline 100 & 200 & 380 & 610 \\
\hline 80 & 280 & 420 & 860 \\
\hline 60 & 320 & 460 & 1000 \\
\hline 40 & 360 & 500 & 1020 \\
\hline 20 & 380 & 540 & 1040 \\
\hline
\end{tabular}

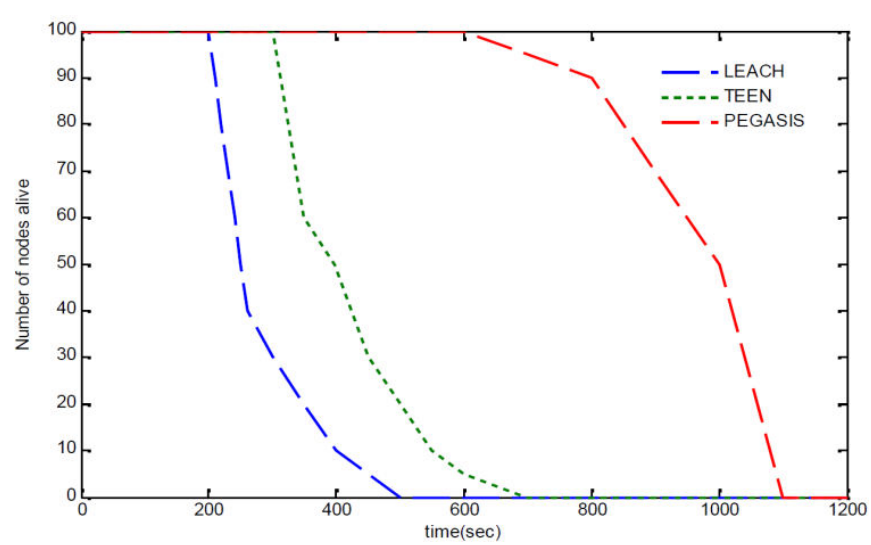

Fig.4. Number of nodes alive

\section{B. Average energy dissipated}

The average energy dissipated statistic depicts the average energy dissipation per node in the network over time while it performs various operations including sending, receiving, sensing, data aggregation, and so on. The average energy dissipation of the protocols under investigation across the number of rounds of operation is shown in this graph. As seen in figure 5, TEEN has a considerably more acceptable energy expenditure curve than LEACH and PEGASIS. Table 2 indicates the average energy dissipated over time for LEACH, TEEN, and PEGASIS protocols.

TABLE 2 AVERAGE ENERGY DISSIPATED

\begin{tabular}{|l|l|l|l|}
\hline TIME & TEEN & LEACH & PEGASIS \\
\hline 100 & 0.0052 & 0.0057 & 0.1056 \\
\hline 200 & 0.2535 & 0.1857 & 0.3556 \\
\hline 300 & 0.8482 & 0.7432 & 0.7254 \\
\hline 400 & 1.5460 & 1.7954 & 1.4365 \\
\hline 500 & 1.7954 & 1.9547 & 1.6543 \\
\hline 600 & 1.9884 & 1.9966 & 1.6968 \\
\hline
\end{tabular}




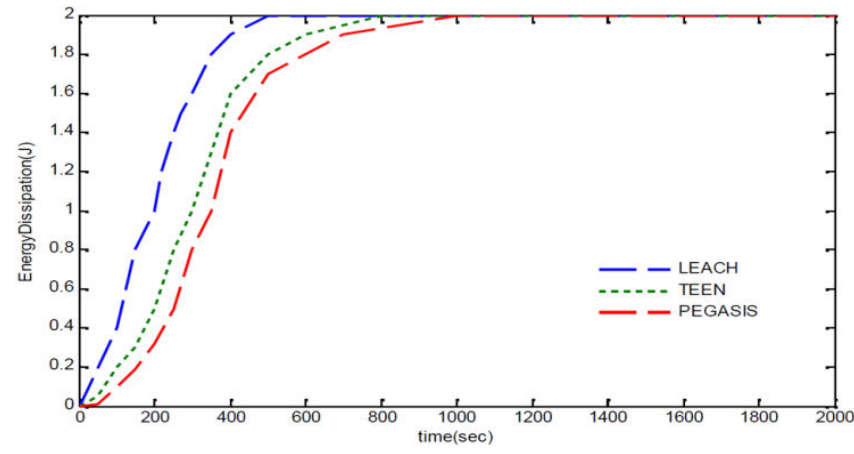

Fig.5. Average energy dissipated

The PEGASIS routing protocol outperforms LEACH by a factor of two when the network range is maintained constant. The energy wasted among all of the sensor nodes is balanced due to the selection of each of the nodes as a head.

It shows how much energy is left in the sensor nodes. Sensor nodes utilize less energy since each cluster's cluster head $(\mathrm{CH})$ gets data from its cluster members. When the packet delivery ratio rises, the node's energy consumption rises as well, but when the transmitting time and distance fall, the sensor nodes' energy consumption rises. As a result, the graph in figure 5 TEEN shows that the network's residual energy is enormous when compared to other protocols like LEACH and PEGASIS.

\section{Operating Nodes per Round}

The performance of these protocols was seen here, with the LEACH protocol having the first dead node after 200 rounds of simulation and total dead nodes after 10 rounds of simulation being 6 . The first dead node in the TEEN protocol occurs after 200 simulation rounds, and there are a total of four dead nodes after 150 simulation rounds. For the PEGASIS protocol, the first dead node occurs after 300 time simulations, and the first 10 rounds take 180 seconds.

PEGASIS starts with the farthest node and builds a chain structure as it moves towards adjacent sensor nodes using a greedy approach. The basic notion is that the nodes communicate with their neighbors, and that once data is collected, it is consolidated in one location before being delivered to the sink node. The network leader is chosen in turns, ensuring that energy is distributed evenly across all nodes. Because energy is distributed evenly, the network's efficiency and endurance are improved.

The size of the clusters and the location of nodes in LEACH vary. Energy consumption increases as the network's node spread changes in range, having a substantial impact on the network's overall performance. Table 3 shows the data values for the number of rounds for the LEACH, TEEN, and PEGASIS processes.
TABle 3 OPERATING NODE PER ROUND

\begin{tabular}{|c|l|l|l|}
\hline $\begin{array}{l}\text { Number of } \\
\text { Rounds }\end{array}$ & TEEN & LEACH & PEGASIS \\
\hline 1 & 224.64 & 173.24 & 223.24 \\
\hline 4 & 251.29 & 173.24 & 223.24 \\
\hline 6 & 279.46 & 199.78 & 249.78 \\
\hline 8 & 309.12 & 257.44 & 307.44 \\
\hline
\end{tabular}

When the transmission range of a sensor network is limited, PEGASIS can significantly extend its lifetime, as demonstrated in figure 6 . The TEEN protocol is ideal for time-critical applications since it is both energy efficient and rapid to reply.

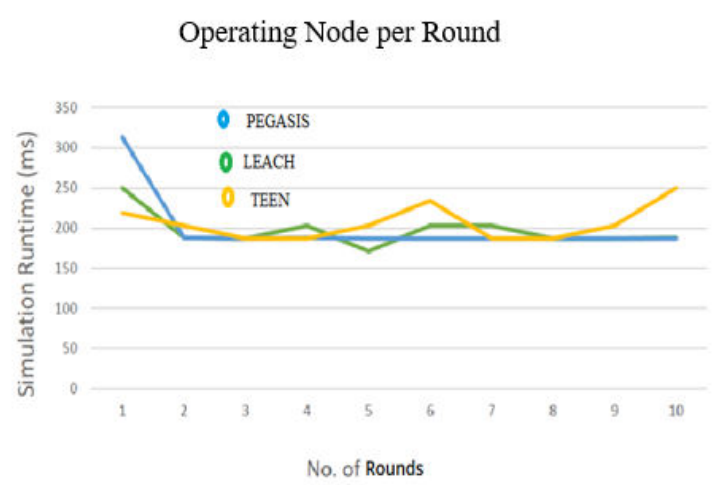

Fig.6. Number of rounds

It also enables the user to tailor the energy usage and precision to the application. The sensor nodes and the base station in TEEN share the identical beginning energy. Data can be sent directly from the base station to all nodes in the network. PEGASIS, on the other hand, assumes that all sensor nodes have the same amount of energy and will die at the same time. The chain may be easily formed using a greedy algorithm because all nodes are stationary and have global knowledge of the network. The chain is created at a node that is far away from the base station. Only one of its neighbors' nodes broadcasts and receives data at a time. The node transfers the token to the leader from both sides of the chain. Each node mixes the supplied data with their own data to construct the chain.

\section{Network lifetime}

TABLE 4 NETWORK LIFETIME

\begin{tabular}{|l|l|l|l|}
\hline Time & LEACH & PEGASIS & TEEN \\
\hline 200 & 79.85 & 90.05 & 84.89 \\
\hline 400 & 81.22 & 87.22 & 85.23 \\
\hline 600 & 87.90 & 96.36 & 89.20 \\
\hline 800 & 83.51 & 88.91 & 82.35 \\
\hline
\end{tabular}

PEGASIS has a shorter data transmission distance between nodes than LEACH. When the network range is kept constant, the PEGASIS routing protocol outperforms LEACH by a factor of two. 


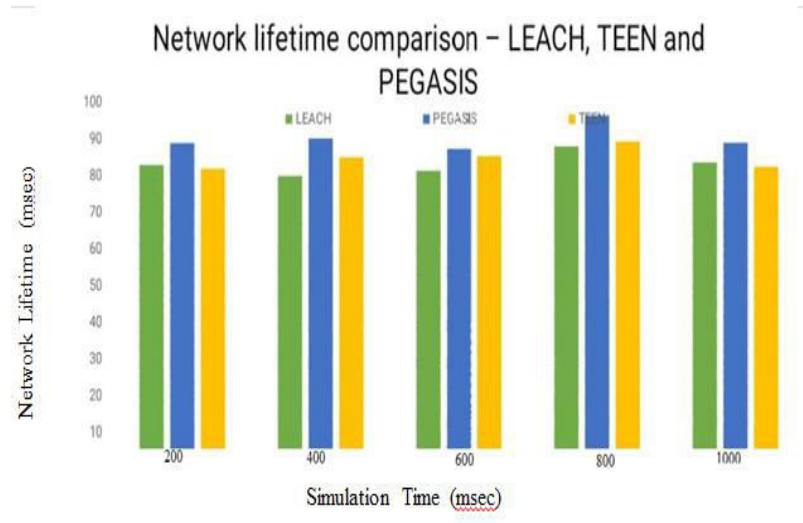

Fig. 7. Three protocols Network lifetime comparison

When it comes to node and network longevity, PEGASIS outperforms LEACH. PEGASIS outlasts LEACH in terms of network longevity. Figure 7 depicts the sensor nodes' network lifetime.

PEGASIS Network has a longer lifespan than LEACH and TEEN. By reducing the overhead of dynamic cluster information, minimizing the sum of distances, and limiting the number of transmissions, PEGASIS outperforms LEACH and TEEN.

TEEN only sends time-critical data, whereas PEGASIS sends data on a regular basis. PEGASIS is also superior to LEACH since it transmits data depending on a threshold value rather than continuously transmitting data. PEGASIS avoids the LEACH clustering overhead, although it does necessitate dynamic topology change because sensor energy is not recorded. PEGASIS adds an inordinate amount of latency to the chain's distant nodes. In PEGASIS, a single leader might become a bottleneck. When compared to the LEACH protocol, PEGASIS extends the network lifetime.

\section{CONCLUSION}

In this work, three common hierarchical routing protocols of wireless sensor networks were analyzed and evaluated. The performance evaluation criteria are based on: number of nodes a live, average energy dissipated, operating nodes per round and network lifetime where these factors have the most effect on the chosen protocols under evaluation. LEACH is not recommended to deploy in large networks, because of the single hop routing technique and the BS with LEACH protocol gets a smaller number of nodes alive than the other two protocols PEGASIS and TEEN. It has a smaller number of nodes alive than the TEEN and PEGASIS protocols. In addition, the network which uses LEACH protocol consumes more energy than the networks using other protocols.

Furthermore, the PEGASIS protocol has more active nodes every round than both the TEEN and LEACH protocols. In TEEN networks, the sensor node is more advanced. Nodes communicate at several levels, providing for maximum efficiency and time savings. It's useful when the environment abruptly changes (it is designed to be responsive to sudden changes in temperature). PEGASIS is the way to go when power is critical and sensor battery life is a big factor. This protocol is useful in large WSNs, when time is not critical and power is important, because the PEGASIS protocol has more network lifetime and less average energy consumptions in the network. Simulation results show that PEGASIS reduces overall energy consumption and improves network lifetime over its comparatives.

This research finding will access the challenge of WSN and overcome the challenge. Hence society's benefit, considering that the network life time the WSN a vital role in science and technology today. The greater demand for the research with a WSN justifies the need for more effective and efficient, WSN protocol with regard to network life time. Other factors that can be investigated further to evaluate and compare the performance of hierarchical routing protocols include fault tolerance, topology change, mobility, cost, environment, scalability, and data fusion, though their impacts are limited and closely related to the investigated WSN application.

\section{ACKNOWLEDGEMENTS}

The authors would like to express sincere thanks for the encouragement and constant support provided by the School of Computing \& Informatics, COET, Dilla University, Dilla, Ethiopia during this work.

\section{REFERENCES}

[1] Shiue, S.M., Huang, L.J., Tsai, W.H. and Chen, Y.L., On Four Metaheuristic Applications to Speech Enhancement-Implementing Optimization Algorithms with MATLAB R2018a. In Proceedings of the 30th Conference on Computational Linguistics and Speech Processing (ROCLING 2018) 2018, 266-275.

[2] Somauroo, A., \&Bassoo, V, Energy-efficient genetic algorithm variants of PEGASIS for 3D wireless sensor networks. Applied Computing and Informatics. 2020

[3] Orngreen, Rikke, and Karin Levinsen. "Workshops as a Research Methodology." Electronic Journal of E-learning 15, no. 1, 2017, pp 70-81.

[4] Khan, A. R., Rakesh, N., Bansal, A., \&Chaudhary, D. K.. Comparative study of WSN protocols (LEACH, PEGASIS and TEEN). Third International Conference on Image Information Processing (ICIIP) 2015, pp. 422-427.

[5] Razaque, Abdul, MusbahAbdulgader, Chaitrali Joshi, FathiAmsaad, and MrunalChauhan. "PLEACH: energy efficient routing protocol for wireless sensor networks." IEEE Long Island Systems, Applications and Technology Conference (LISAT), 2016, pp. 1-5.

[6] Saini, Parul, and Ajay K. Sharma. "E-DEECenhanced distributed energy efficient clustering scheme for heterogeneous WSN." First international conference on parallel, distributed 
and grid computing (PDGC 2010). 2010 IEEE.

[7] K S Ananda Kumar., and R. Balakrishna. "Evaluation of Energy Consumption using Receiver-Centric MAC Protocol in Wireless Sensor Networks." International Journal of Electrical and Computer Engineering (IJECE) 8, no. 1, 2018, pp 87-93.

[8] Prasad, A. Y., and R. Balakrishna. "Implementation of optimal solution for network lifetime and energy consumption metrics using improved energy efficient LEACH protocol in MANET." Telkomnika 17, no. 4 2019, pp 17581766.

[9] Hamdy H. El-Sayed, Hassan Shaban Hassan; Performance Comparison of Various Hierarchical WSN Routing Protocols, Int. J. Advanced Networking and Applications, Volume: 11 Issue: 02, 2019, pp 4218-4223.

[10] Sahar Alsafi, Samani A. Talab; Implementation of DEEC, DDEEC, EDEEC and TDEEC Protocols using MATLAB in Wireless Sensor Network; Int. $J$. Advanced Networking and Applications, Volume: 12 Issue: 03, 2020, pp 4596-4600.

\section{Author's Biography}

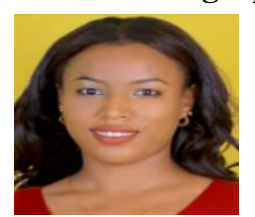

Hebron Hailu is working as lecturer at Hawassa College of Teacher Education. She received her BSC degree in Computer Science and MSC degree in Computer Science and Networking from Hawassa and Dila University in 2015 and 2021 respectively. Her research interests are WSN, IoT and Data Science.

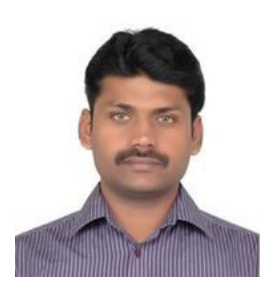

Dr. K S Ananda Kumar received his Ph.D. degree in Computer \& Information Science from Visvesvaraya Technological University, Belagavi, Karnataka, India. His research interests include wireless sensor networks, IOT, Machine learning, Data Science.

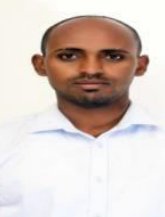

Mr. Girmay Tekle was born in Zalambesa, Ethiopia. He received the B.Sc. degree in computer science from Aksum University, Ethiopia, the M.Sc. degree in Informatics from Silesian University of Technology, Poland, in 2012 and 2016, respectively. In addition to that he has got Blockchain Developer Mastery Award (2018) certificate form IBM Digital nation Africa in 2019. In August of 2012, he joined Dilla University as a Graduate Assistant II. He then has been served with different positions and currently working as Lecturer, School dean of computing and Informatics School and Research Adhoc Committee member of Engineering and Technology college. In June 2021, he has won a research grant of Dilla University to conduct research in illegal building detection using Machin learning. His research interests include IoT
Network, Blockchain, Data Security, Cloud Computing, Network, Machine Learning, solving Computer Vision Problems with deep learning approach

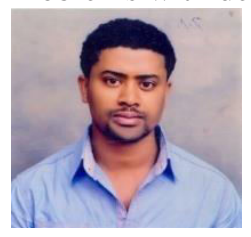

Mr. Sisay Muleta Hababa currently working as Lecturer and Researcher in School of Computer \& Informatics, College of Engineering \& Technology, Dilla University, Dilla, Ethiopia. He received his B.Sc degree from Dilla University, Dilla, Ethiopia. He received his M.Sc in Information and communication Engineering from University of Electronic Science and Technology of China, Chengdu, China. His research interests are Networks, cloud computing, IoT and Data science. 